\title{
X-ray photoelectron spectroscopy study of low-temperature molybdenum oxidation process
}

\author{
S. I. Castañeda, I. Montero, ${ }^{\text {a) }}$ J. M. Ripalda, N. Díaz, L. Galán, and F. Rueda \\ Departamento de Fisica Aplicada C-XII, Universidad Autónoma de Madrid, \\ Cantoblanco, 28049-Madrid, Spain
}

(Received 5 August 1998; accepted for publication 8 March 1999)

\begin{abstract}
The low-temperature oxidation during deposition by evaporation of molybdenum thin films has been investigated. Analysis by x-ray photoelectron spectroscopy and x-ray diffraction reveals that small differences in the substrate temperature during deposition may give rise to important changes in the final composition and structure of the molybdenum oxide. Changes in binding energy and line shape of the Mo $3 d_{5 / 2}-$ Mo $3 d_{3 / 2}$ doublet attributed to oxygen incorporation have been studied. Two principal steps can be distinguished, with a transition temperature of $\sim 310^{\circ} \mathrm{C}$. Up to substrate temperatures of $\sim 310^{\circ} \mathrm{C}$, the superficial Mo remains almost unaffected, with some oxygen dissolved. At $\sim 310^{\circ} \mathrm{C}$, mixing of $\mathrm{Mo}^{0}$ metal and molybdenum oxide $\left(\mathrm{Mo}^{\delta+} 0<\delta<4\right)$ clusters or islands is observed. Finally, above this temperature a surface layer of molybdenum oxide, $\mathrm{Mo}^{6+}$, is formed. In addition, an abrupt change in $d_{100}$ interplanar parameter of Mo is observed. (C) 1999 American Institute of Physics. [S0021-8979(99)00312-6]
\end{abstract}

\section{INTRODUCTION}

The oxidation process of molybdenum surfaces has received considerable attention because of its important technological applications. ${ }^{1-7}$ Molybdenum oxides are excellent catalysts for several catalytic reactions such as the partial oxidation of organic molecules. ${ }^{8}$ Sulfidation of molybdenum oxides is of special interest because of their catalyst applications. ${ }^{9} \alpha-\mathrm{MoO}_{3}$ seems to be particularly interesting for applications in solid state batteries and display systems, because of its two-dimensional layered structure..$^{10,11} \mathrm{Al}$ though molybdenum oxides are used in a variety of applications, the nature and catalytic properties of these materials are not well understood as yet. The hydrogen reduction of $\mathrm{Mo}^{6+}$ states produces $\mathrm{MoO}_{2}$ as well as a new state $\left(\mathrm{Mo}^{\delta+} 0<\delta<4\right)$ that could be the active site responsible for catalytic reactions. $^{12}$

Interaction of oxygen with molybdenum single crystal surfaces at high temperatures can produce metal oxidation states corresponding to amorphous $\mathrm{MoO}_{3}$ and intermediate oxides, in addition to ordered $\mathrm{MoO}_{2} \cdot{ }^{13}$ However, $\mathrm{Mo}^{6+}$ states present during the oxidation of Mo (100) have also been associated to the formation of surface polymolybdate species on the oxidized layer. ${ }^{14}$ In the present work, x-ray photoelectron spectroscopy (XPS) is used to examine the chemical state of Mo deposited at medium substrate temperatures $\left(200-450^{\circ} \mathrm{C}\right)$ by evaporation with an electron gun. A quantitative analysis of the XPS data yields information about the different elements and the structure of the oxidized layer. Complementary results have been obtained by $\mathrm{x}$-ray diffraction (XRD).

\footnotetext{
a) Also at: Instituto de Ciencia de Materiales de Madrid, CSIC, Campus de la Universidad Autónoma de Madrid, Cantoblanco, 28049-Madrid, Spain; Electronic mail: imontero@icmm.csic.es
}

\section{EXPERIMENT}

Molybdenum thin films were deposited in a vacuum chamber with a base pressure of $\sim 10^{-8}$ Torr, by evaporation with a $4 \mathrm{kV}$ dc $350 \mathrm{~mA}$ electron gun with magnetic deflection and a water-cooled copper crucible onto clean Corning glass substrates. These glass substrates were heated by halogen lamps of $500 \mathrm{~W}$ mounted at the top of the preparation chamber, to temperatures in the range $200-450{ }^{\circ} \mathrm{C}$. The pressure was $\sim 10^{-6}$ Torr during deposition. The film thickness was measured with a quartz crystal monitor during deposition and with a stylus type apparatus, Talystep Taylor\&Hobson, U.K. after deposition. The deposition rate range was between 1.5 and $2.5 \mathrm{~nm} / \mathrm{min}$ and deposition time about $3 \mathrm{~h}$.

XPS analysis was performed at room temperature with a VGS ESCALAB 210 instrument using nonmonochromatic $\operatorname{Mg} K \alpha$ X-radiation $(h \nu=1253.6 \mathrm{eV})$, which was operated in the constant-pass energy mode. The resolution of the electron-energy analyzer was about $0.5 \mathrm{eV}$. First, the samples were introduced into the spectrometer about $5 \mathrm{~min}$ after being taken out of the deposition system. The samples were sputter cleaned by $2 \mathrm{kV} \mathrm{Ar}^{+}$ion bombardment performed with a differentially pumped ion gun, at 5 $\times 10^{-7}$ Torr in the XPS analyzer chamber. The plasma chamber of the ion gun was always operated under dynamic gas flow conditions with a continuous stream of fresh gas.

After ion bombardment, the carbon atomic fraction of the samples remains nearly constant, at about 0.03 . In some samples, XPS analysis also revealed the incorporation of $\mathrm{Na}$ $(<5 \%)$ from the glass substrate into the molybdenum oxide during the deposition process.

XRD patterns were obtained with a Siemens D-5000 diffractometer. The radiation used was $\operatorname{Cu} k \alpha(\lambda=1.5406 \AA)$, in a $\theta-2 \theta$ configuration, the full-width at half-maximum 


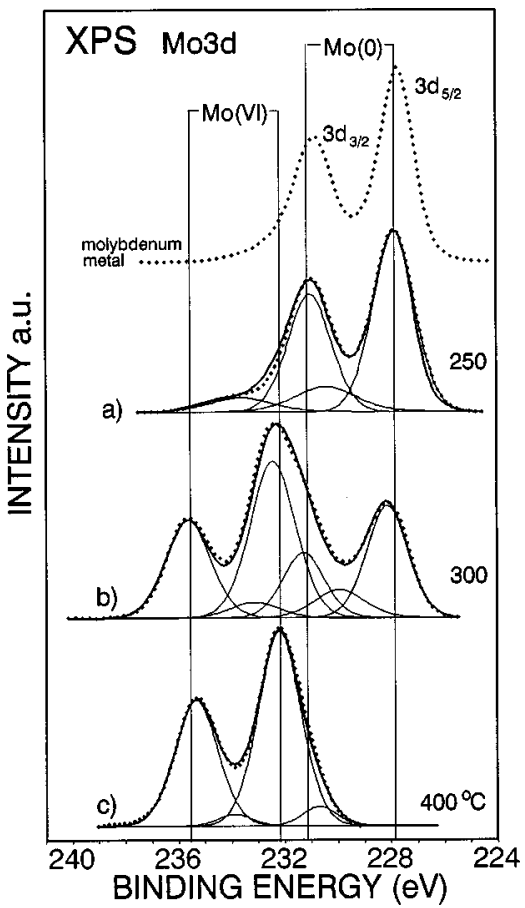

FIG. 1. XPS spectra of the Mo $3 d$ core levels for substrate temperatures $T_{s}=250,300$, and $400^{\circ} \mathrm{C}$, curves (a), (b), and (c), respectively. Pure Mo foil has been also included for comparison. The principal doublets of $\mathrm{Mo}^{\circ}$ and $\mathrm{Mo}^{6+}$ states are marked.

(FWHM) and the intensity of the peaks were computed by regular and split Pearson refinement. ${ }^{15}$

\section{RESULTS AND DISCUSSION}

The dependence of the structure and composition of the samples on substrate temperature, $T_{s}$ in the $200-450{ }^{\circ} \mathrm{C}$ range, has been studied. XPS technique permits to identify elements and their chemical states present in the outermost $\sim 5 \mathrm{~nm}$ of the surface of the samples. Figure 1 shows a XPS spectral representative series of Mo $3 d$ core levels for three different deposition temperatures: 250,350 , and $400^{\circ} \mathrm{C}$, labeled spectra $a, b$, and $c$. A pure Mo metal spectrum has also been included for comparison. The Mo $3 d$ core levels of the metal split into the $3 d_{3 / 2}$ and $3 d_{5 / 2}$ spin-orbit partners (split by $\delta_{S O}=3.15$ and $3.20 \mathrm{eV}$, for molybdenum and molybdenum oxide, respectively, which reflects very closely the statistical intensity ratio $I_{S O}=I_{3 / 2} / I_{5 / 2}=2 / 3$ ). The Mo $3 d$ line of the metal shows the typical asymmetry produced by lowenergy excitations. The resulting shape and chemical shifts of the Mo $3 d_{5 / 2}$ core level peaks of the sample may be the main effects of substrate temperature $T_{s}$. The broadening of the signal towards higher binding energies is due mainly to electrons emitted from the $\mathrm{MoO}_{x}$ phases.

In order to perform a quantitative analysis of the chemical species, the spectra were smoothed and their background was subtracted by the usual procedures, ${ }^{16,17}$ and fitted in terms of several doublets maintaining $\delta_{S O}$ and $I_{S O}$ as constants. The intensity was measured as the peak area. Typical results of the line shape analysis are also shown in Fig. 1. Solid lines show the individual components of the mathematical fit. The principal doublets obtained in the math-

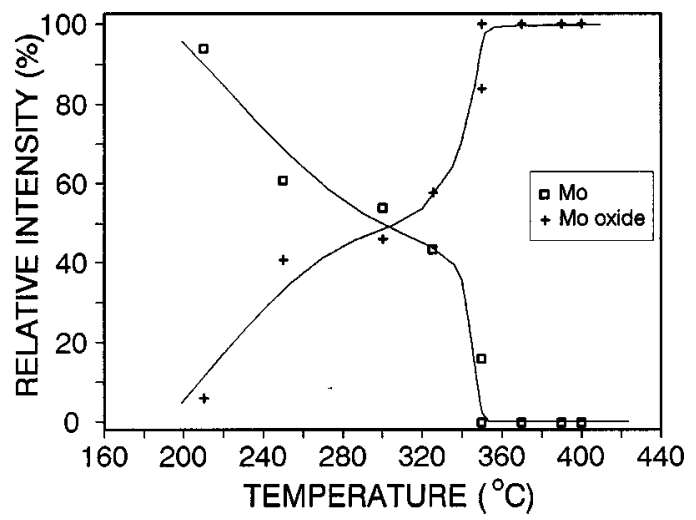

FIG. 2. Relative intensity of the $\mathrm{Mo}(0)$ and $\mathrm{Mo}(\mathrm{VI})$ doublet peaks as a function of substrate temperature.

ematical fits are $\operatorname{Mo}(0)$ and $\mathrm{Mo}(\mathrm{VI})$, as labeled in Fig. 1, which correspond to $\mathrm{Mo}^{0}$ and $\mathrm{Mo}^{6+}$ states, respectively. The other states $\mathrm{Mo}^{\delta+}$ for $0<\delta<6$ contribute less than $10 \%$. We can observe that for $T_{s}<310^{\circ} \mathrm{C}$, the $\operatorname{Mo}(0)$ doublet is the most intense. However, close to and above this temperature, more than one phase exists, the spectra present a noticeable broad and asymmetrical shape and a shift to higher binding energies. $\mathrm{MoO}_{2}$ can be present when the thickness of oxide layer is between 0.6 and $0.8 \mathrm{~nm} .{ }^{14}$

Figure 2 shows the qualitative evolution of the relative intensity of the $\mathrm{Mo}(0)$ and $\mathrm{Mo}(\mathrm{VI})$ peaks as a function of the substrate temperature. The low contribution of the $\mathrm{Mo}^{\delta+}$ states is omitted. ${ }^{12} \mathrm{We}$ can observe that up to $T_{s}<310^{\circ} \mathrm{C}$ the percentage of the $\operatorname{Mo}(0)$ doublet decreases as a function of temperature from about $95 \%$ to $50 \%$, and disappears for $T_{s}$ $>310^{\circ} \mathrm{C}$. These results may indicate that initially, for $T_{s}$ $<310^{\circ} \mathrm{C}$, the surface of the sample is formed by a mixture of Mo and molybdenum oxide, but for $T_{s}>310^{\circ} \mathrm{C}$ the oxide clusters or islands coalesce, forming a uniform oxide across the whole surface. Thus, for $T_{s}>310^{\circ} \mathrm{C}$ the spectra indicated the formation of $\mathrm{Mo}^{6+}$ oxidation state, which can be attributed to an internal electron transfer from metal orbitals to oxygen by thermal ionization. Moreover, we have observed that the surface oxidation of molybdenum is not affected by the deposition rate of the coatings.

The energy difference between the $\mathrm{O} 1 s$ and Mo $3 d_{5 / 2}$ core level centroids, $\Delta\left(\mathrm{O} 1 s-\mathrm{Mo} 3 d_{5 / 2}\right)$, has been used to characterize the Mo-O bonds, although the interpretation of $\Delta\left(\mathrm{O} 1 s-\mathrm{Mo}_{3} d_{5 / 2}\right)$ in terms of structural variations in the molybdenum oxide network may seem ambiguous considering the complex line shapes and scape depth differences involved. The electron scape depth ratio for Mo $3 d$ and $\mathrm{O} 1 s$ photoelectrons, $\lambda_{\mathrm{Mo}} / \lambda_{\mathrm{O}}$, may be estimated as $\lambda_{\mathrm{Mo}} / \lambda_{\mathrm{O}}$ $=\left[E_{k}(\mathrm{Mo}) / E_{k}(\mathrm{O})\right]^{1 / 2} \cong 1.19$, where $E_{k}(\mathrm{Mo})$ and $E_{k}(\mathrm{O})$ are the kinetic energies of the Mo $3 d$ and $\mathrm{O} 1 s$ photoelectrons, respectively. The $\mathrm{O} 1 s$ and Mo $3 d$ core level spectra are not probing exactly the same region of the oxide. However, considering the relatively small difference between the scape depths, we can assume that Mo $3 d$ signal is integrated along an oxide depth similar to the $\mathrm{O} 1 \mathrm{~s}$ signal. The $\Delta(\mathrm{O} 1 \mathrm{~s}$ - Mo $3 d$ ) shows the relative O-Mo chemical shifts, and, according to the basic principles of the XPS analysis, they should be a measure of the ionicity of the bond and the 
charge transfer. On the other hand, the chemical shift of the Mo $3 d_{3 / 2}$ centroid should be approximately proportional to the overall composition $\mathrm{O} / \mathrm{Mo}$ of the near-surface region probed by XPS. ${ }^{18}$ Also, the $\Delta\left(\mathrm{O} 1 s-\mathrm{Mo} 3 d_{5 / 2}\right)$ is insensitive to energy reference problems such as surface charging.

Figure 3 shows $\Delta\left(\mathrm{O} 1 s-\mathrm{Mo} 3 d_{5 / 2}\right)$ values for several deposition temperatures. The solid marks indicate the more intense peaks for each $T_{s}$. For $T_{s}<310^{\circ} \mathrm{C}$ the energy difference remains almost unchanged compared to that of the clean Mo metal, $303.2 \mathrm{eV} .{ }^{19}$ Close to $310^{\circ} \mathrm{C}$, apart from the $\mathrm{Mo}^{0}$ state, another doublet peak is detected at $299.8 \mathrm{eV}$, which can be associated to $\mathrm{Mo}^{4+}$ states, although their intensity is small, as explained below. Finally, for $T_{s}>310^{\circ} \mathrm{C}$, $\Delta\left(\mathrm{O} 1 s-\mathrm{Mo} 3 d^{5 / 2}\right)$ becomes $\sim 297.5 \mathrm{eV}$. These results are related to the chemical shift of Mo toward higher binding energies during the oxidation process. No chemical shift of the Mo $3 d$ takes place before the conversion of chemisorbed oxygen into oxide state.

The average near-surface composition, O/Mo atomic ratio, has been computed from the XPS peak intensities and the corresponding XPS sensitivity factors of the Mo $3 d$ and $\mathrm{O} 1 s$ core levels. ${ }^{20}$ Since XPS intensities are proportional to element concentration (number of atoms per analyzed volume) and intensity ratios were used for the quantitative analysis, the results refer to atomic ratios, i.e., the number of atoms of a chemical species divided by the total number of atoms in the analyzed volume. Figure 4 shows the substrate temperature dependence of surface concentration ratio O/Mo. There is an initial region where the composition remains practically constant with the temperature up to $\sim 310^{\circ} \mathrm{C}$, followed by an abrupt increase until the composition $\mathrm{O} / \mathrm{Mo} \approx 3$ is obtained. The insert of Fig. 4 shows the plot $\ln (\mathrm{O} / \mathrm{Mo})$ versus $1 / T$. The results present an Arrhenius-type behavior, with an activation energy of $0.13 \mathrm{eV}$ for the process. This low activation energy can be attributed to the presence of high density of grain boundaries with high-diffusivity pathways. For instance, in the case of flash-evaporated molybdenum oxide at $T_{s}=250{ }^{\circ} \mathrm{C}$, the activation energy obtained after annealing treatments is $0.6 \mathrm{eV} .^{21}$ It is well known that at high temperatures, mass transport is thought to involve diffusion driven

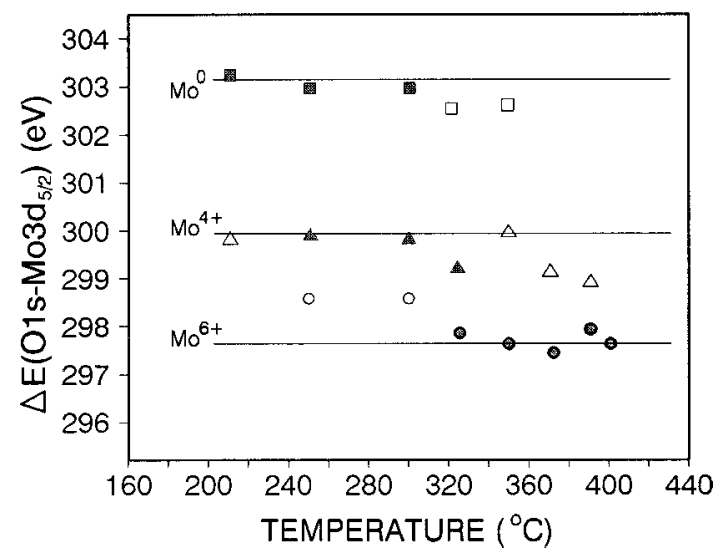

FIG. 3. Binding energy difference between the $\mathrm{O} 1 \mathrm{~s}$ and Mo $3 d_{5 / 2}$ core level peaks as a function of substrate temperature. Lines are the reference values for the chemical species indicates (Ref. 18).

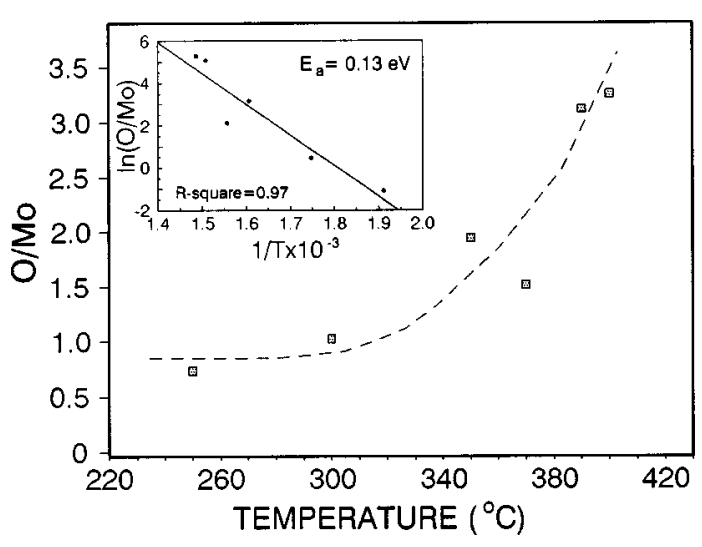

FIG. 4. O/Mo atomic ratio surface composition as a function of substrate temperature. The insert shows the Arrhenius plot and a linear fit.

across the oxide film by a chemical gradient, as described by the Wagner mechanism for parabolic oxidation. However, at low temperatures, an electric field across the film, resulting from chemisorption of oxygen ions on the oxide surface, is also taken into account to explain the continuous reaction. ${ }^{22}$ The evolution of the as-deposited samples during several days of air exposure has also been studied. In all cases the carbon and oxygen contents drastically increase with time: the value of carbon content, for instance, can reach 40 at. \%.

The general features of the XRD patterns of the samples indicate only a polycrystalline Molybdenum with $\sim 20 \mathrm{~nm}$ grain size. The molybdenum oxide consists of $\mathrm{MoO}_{6}$ octahedra linked together by bridging oxygen atoms. Although the $\mathrm{O}-\mathrm{Mo}-\mathrm{O}$ bond angle within a given octahedron or tetrahedron is not distorted, the Mo-O-Mo bond angle is known to vary over a significant range in $\mathrm{MoO}_{x}$ oxides. ${ }^{23}$ Analysis of the XRD patterns of these samples shows changes in (110) interplanar distance, $d_{110}$, with substrate temperature as shown in Fig. 5. Surprisingly, an abrupt increase of $d_{110}$ at $T_{s} \approx 310^{\circ} \mathrm{C}$ has been observed. This variation reflects corresponding changes in the a lattice parameter, possibly due to changes in the degree of oxygen concentration dissolved in the bulk, which could indicate the presence of the $\mathrm{MoO}_{x}$ phases for $T_{s}>310^{\circ} \mathrm{C}$. It is known that there is an increase in the lattice parameters as the oxygen concentration increases. Therefore, we can assume that oxide thin films in the first oxidation steps $\left(T_{s}<310^{\circ} \mathrm{C}\right)$ were composed of oxygen

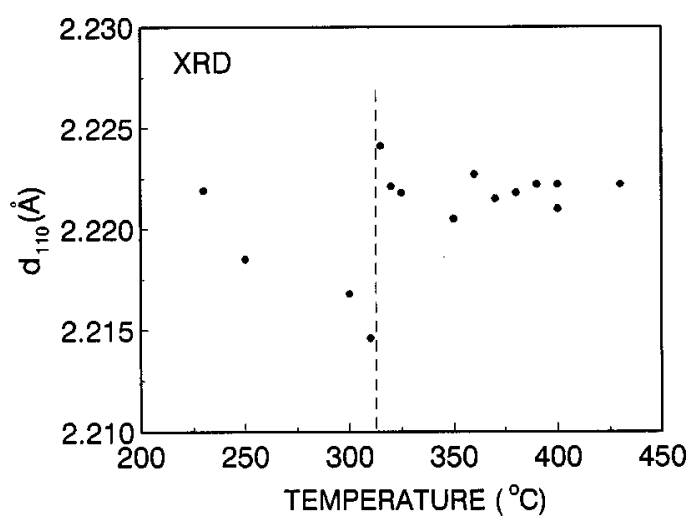

FIG. 5. $d_{110}$ as a function of the substrate temperature. 
dissolved in the molybdenum lattice. Enhanced oxygen diffusion by vacancies and grain boundaries is very likely and would help to explain the rapid incorporation of oxygen at these low temperatures. It has been established ${ }^{24}$ that the oxidation states of transition metal ions and the oxygen vacancy structures around them exert a strong influence on the selectivity of catalyzed oxidation process, as the present results seem to confirm.

\section{CONCLUSIONS}

The oxygen incorporation during the film deposition at $10^{-6}$ Torr depends drastically on the substrate temperature. The evolution of the intensities of the Mo $3 d$ and $\mathrm{O} 1 s$ core level peaks, associated with the oxide formed, as a function of the substrate temperature provides evidence of the formation of structurally distinct oxides. Mo remains unaffected up to temperatures of $310^{\circ} \mathrm{C}$ with some oxygen dissolved. Above this temperature, $\mathrm{Mo}^{6+}$ species are detected. It is concluded from this work that the interaction of oxygen with molybdenum proceeds according to a two-stage process with a transition temperature of about $310^{\circ} \mathrm{C}$.

\section{ACKNOWLEDGMENTS}

This work has been supported by CICYT, project ESP96-0956, DGYCIT: Grant no. PB94-0201 and Comunidad de Madrid no. Reg. Exp.: 07N/0044/1998 of Spain.

\footnotetext{
${ }^{1}$ E. Bauer and H. Poppa, Surf. Sci. 88, 31 (1979).

${ }^{2}$ Yu. V. Plyuto, I. V. Babich, I. V. Plyuto, A. D. Van Langeveld, and J. A. Moulijn, Appl. Surf. Sci. 119, 11 (1997).
}

${ }^{3}$ C. Zhang, M. A. Van Hove, and G. A. Somorjai, Surf. Sci. 149, 326 (1985).

${ }^{4}$ L. Soriano, J. F. Trigo, L. Galán, and F. Rueda, Surf. Interface Anal. 19, 553 (1992).

${ }^{5}$ W. Dong and B. Dunn, J. Non-Cryst. Solids 225, 135 (1998).

${ }^{6}$ M. Di Giulio, D. Manno, and A. Tepore, Phys. Status Solidi A 168, 249 (1998)

${ }^{7}$ Z. Xiang Lu, Y. Qing Li, and S. Xie Qi, Appl. Catal. 56, 207 (1989).

${ }^{8}$ L. E. Firment and A. Ferretti, Surf. Sci. 129, 155 (1983).

${ }^{9}$ P. A. Spevack and N. S. McIntyre, J. Phys. C 97, 11031 (1993).

${ }^{10}$ P. A. Spevack and N. S. McIntyre, J. Phys. C 97, 11020 (1993).

${ }^{11}$ J. O. Besenhard, J. Heydecke, E. Wudy, H. P. Fritz, and W. Foag, Solid State Ionics 8, 61 (1983).

${ }^{12}$ J. G. Choi and L. T. Thompson, Appl. Surf. Sci. 93, 143 (1996).

${ }^{13}$ C. Zhang, M. A. Van Hove, and G. A. Somorjai, Surf. Sci. 149, 326 (1985).

${ }^{14}$ G. H. Smudde, Jr. and P. C. Stair, Surf. Sci. 317, 65 (1994).

${ }^{15}$ R. A. Young and D. B. Wiles, J. Appl. Crystallogr. 15, 430 (1982).

${ }^{16}$ D. A. Shirley, Phys. Rev. B 5, 4709 (1972).

${ }^{17}$ H. E. Bishop, Surf. Interface Anal. 3, 272 (1981).

${ }^{18}$ L. Galán, P. Prieto, C. Morant, L. Soriano, J. M. Sanz, E. Elizalde, and F. Rueda, Surf. Interface Anal. 19, 205 (1992).

${ }^{19}$ NIST x-ray photoelectron spectoscopy database, National Institute of Standard and Technology, Gaithersburg, MD (1997).

${ }^{20}$ XPS sensitivity factors Data Sheet supplied by Leybold-Heraeus with a LH 10 ESCA spectrometer, 1985, corrected for transmission function of VGS ESCALAB instrument.

${ }^{21}$ C. Julien, A. Khelfa, O. M. Hussain, and G. A. Nazri, J. Cryst. Growth 156, 235 (1995).

${ }^{22}$ A. T. Fromhold, Jr., Theory of Metal Oxidation, Series Defects un Cristalline Solids, Vol. 9 (North-Holland, Amsterdam, 1976), p. 106.

${ }^{23}$ Inorganic Solids; An Introduction to Concepts in Solid-State Structural Chemistry, edited by D. M. Adams (Wiley, New York, 1973), Chap. 9, p. 320.

${ }^{24}$ J. L. Callahan, R. K. Graselli, E. C. Milberger, and H. A. Strecker, Ind. Eng. Chem. Products Res. Develop. 9, 134 (1979) 